\title{
Associação entre expectativa de autoeficácia e adesão ao tratamento antirretroviral em adultos com HIV
}

Association between expectation of self-efficacy and adherence to antiretroviral treatment in adults with HIV

Asociación entre expectativa de autoeficacia y adhesión al tratamiento antirretroviral en adultos con VIH

\section{Resumo}

Objetivo: Avaliar a associação entre a expectativa de autoeficácia e a adesão ao tratamento antirretroviral em adultos com HIV. Método: Estudo transversal desenvolvido em serviço especializado no Sul do Brasil com 156 participantes. Foi aplicada a Escala de Expectativa de Autoeficácia para Seguir Prescrição Antirretroviral (EEASPA) e o Questionário para a Avaliação da Adesão ao Tratamento Antirretroviral em pessoas com HIV/AIDS (CEAT-VIH). Análise de correlação de Spearman. Resultados: As experiências de aborrecimento, discriminação, rejeição, insegurança em saber como as pessoas (de convívio próximo) vão reagir ao saber do diagnóstico, nervosismo, irritação, ingestão de grande quantidade de comprimidos e outros efeitos adversos causados pela medicação apresentaram correlação significativa com a adesão global. Conclusão: As experiências negativas com o uso dos medicamentos e aquelas vivenciadas nas relações sociais apresentaram menores médias para autoeficácia e maior correlação entre a autoeficácia e adesão, indicando a necessidade de desconstrução do estigma.

Palavras-chave: HIV; Síndrome de imunodeficiência adquirida; Autoeficácia; Terapia antirretroviral de alta atividade; Adesão à medicação.

\footnotetext{
Abstract

Objective: To evaluate the association between the expectation of self-efficacy and adherence to antiretroviral treatment in adults with HIV. Method: Cross-sectional study developed in a specialized service in southern Brazil with 156 participants. The Self-Efficacy Expectation Scale for Following Antiretroviral Prescription and the Assessment of Adherence to Antiretroviral Therapy Questionnaire (CEAT-VIH) were applied. Spearman correlation analysis. Results: The experiences of annoyance, discrimination, rejection, insecurity due to the reaction of other
} 
people (in close contact) due to the diagnosis. Additionally, nervousness, irritation, ingestion of large amounts of pills and other adverse effects caused by the medication were found to be significantly correlated with overall adherence. Conclusion: Negative experiences with the use of medications and those experienced in social relationships had lower means for self-efficacy and greater correlation between self-efficacy and adherence, indicating the need to deconstruct the stigma.

Keywords: HIV; Acquired immunodeficiency syndrome; Self efficacy; Antiretroviral therapy, Highly active; Medication adherence.

\section{Resumen}

Objetivo: Evaluar la asociación entre la expectativa de autoeficacia y la adhesión al tratamiento antirretroviral en adultos con VIH. Método: Estudio transversal desarrollado en servicio especializado en el Sur del Brasil con 156 participantes. Fue aplicada la "Escala de Expectativa de Autoeficácia para Seguir Prescrição Antirretroviral" (EEASPA) y el Cuestionario para la Evaluación de la Adhesión al Tratamiento Antiretroviral (CEAT-VIH). Análisis de la correlación de Spearmen. Resultados: Las experiencias del aborrecimiento, discriminación, rechazo, inseguridad en saber cómo las personas (del convivio próximo) van a reaccionar al saber del diagnóstico, nerviosidad, irritación, ingesta de gran cantidad de comprimidos y otros efectos adversos por la medicación presentaran correlación significativa con la adhesión global. Conclusión: Las experiencias negativas con el uso de los medicamentos y las vivencias en las relaciones sociales presentaran menores medias para autoeficacia y mayor correlación entre la autoeficacia y adhesión, indicando la necesitad de desconstrucción del estigma.

Palabras clave: VIH; Síndrome de inmunodeficiencia adquirida; Autoeficacia; Terapia antirretroviral altamente activa; Cumplimiento de la medicación.

\section{Introdução}

A adesão à terapia antirretroviral (TARV) para o controle da infecção pelo vírus da imunodeficiência humana (HIV) foi indicada pelo Programa Conjunto das Nações Unidas sobre HIV/AIDS (UNAIDS) como uma das metas para ser alcançada até 2020. Esse programa previa que 90\% das pessoas que vivem com HIV descobrissem seu diagnóstico, 90\% das diagnosticadas recebessem o tratamento adequado e 90\% das que estão em tratamento alcançassem o nível indetectável para sua carga viral (CV) (Dave et al., 2019). Esse panorama aponta a importância da adesão à TARV e da avaliação deste processo (Souza et al., 2019), para se eleger estratégias de intervenção e monitoramento (Loch et al., 2020; Musayón-Oblitas et al., 2020; Zuge et al., 2020) e melhorar os resultados clínicos do paciente (Cardoso et al., 2019).

O Brasil, como um país que adotou política pública de acesso universal aos antirretrovirais para HIV, proporcionou melhora nos resultados clínicos dos pacientes, com visível declínio nos casos de mortalidade e morbidade e melhoria da qualidade de vida dessas pessoas. Entretanto, ainda se faz necessário elucidar fatores intervenientes no processo de adesão terapêutica, uma vez que estudo com 120 pacientes evidenciou a prevalência de não adesão de 43,3\%. Neste estudo a educação formal de pessoas com ensino superior, foi a variável sociodemográfica relacionada àqueles que apresentaram maior adesão. Também evidenciaram que indivíduos sintomáticos são mais aderentes ao tratamento (Velame et al., 2020).

Outrossim, devido a resposta social para a epidemia do HIV, envolta por estigmas e preconceitos, outros fatores podem influenciar na vida dessa população e afetar a sua autoestima, que poderá ficar prejudicada, como encontrado em $41,1 \%$ $(n=108)$ das pessoas com HIV em estudo do norte do Brasil. Este estudo indicou que o medo de morrer ou da revelação do diagnóstico para outras pessoas, bem como a solidão, vão influenciar na autoestima e a culpa vai influenciar na expectativa de futuro (Patrício et al, 2019). Estes são sentimentos que podem gerar e ausência de apoio familiar e social como mais um dos fatores que interferem na adesão, além das reações adversas à medicação (Santos et al., 2020).

Diante deste fenômeno multifacetado, ainda se tem a questão da expectativa de autoeficácia (EAE) em aderir à TARV. A EAE pode ser definida com base no julgamento do sujeito acerca de sua habilidade para desempenhar com sucesso um padrão específico de comportamento, como por exemplo: tomar medicamentos conforme a prescrição, ter convicção na capacidade pessoal de controlar as circunstâncias que possam dificultar esse comprometimento, mantendo nível de motivação e confiança (Bandura, 1977), além de preocupar-se com o tratamento, influenciando de forma positiva na sua autoeficácia (Amirkhanian et al., 2018). 
A EAE e a adesão podem ser influenciadas de forma negativa pelos efeitos adversos dos medicamentos e circunstâncias de cunho social. Usualmente, essas circunstâncias estão associadas com a preocupação em ser visto tomando a medicação para o HIV por quem não conhece seu diagnóstico e isso explica quase toda a associação entre estigma e adesão (Drachler et al., 2016; Seghatol-Eslami et al., 2017; Zhang et al., 2016).

Diante disso, tem-se como objetivo avaliar a associação entre a expectativa de autoeficácia e a adesão ao tratamento antirretroviral em adultos com HIV.

\section{Método}

Este estudo de delineamento transversal está apoiado na perspectiva cognitivo-social e no referencial teórico da expectativa de autoeficácia (Bandura, 1977). Foi desenvolvido no ambulatório de doenças infecciosas de um hospital universitário do sul do Brasil. Os critérios de inclusão foram: adultos com idade igual ou superior a 18 anos de ambos os sexos em uso de medicamentos antirretrovirais há pelo menos três meses. Os critérios de exclusão foram: estar em regime penitenciário e mulheres em período gestacional. A partir do cálculo amostral, a população mínima esperada era de 147. A coleta de dados ocorreu no período de julho de 2016 a agosto de 2018 e encerrou-se com 156 participantes.

Para a avaliação da adesão ao tratamento antirretroviral, utilizou-se o Cuestionario para la Evaluación de la Adhesión al Tratamiento Antiretroviral (CEAT-VIH), composto por 20 questões que contemplam a conformidade com o tratamento, fatores que modulam a adesão, a interação entre profissional de saúde e o usuário, crenças quanto ao esforço para realizar o tratamento e avaliação dos efeitos colaterais. O grau de adesão global ao tratamento antirretroviral é classificado em adesão “estrita", "insuficiente" e "inadequada" e a pontuação varia de 17 a 89 pontos (Remor et al., 2007).

Para calcular a expectativa de autoeficácia, utilizou-se a Escala de Expectativa de Autoeficácia para Seguir Prescrição Antirretroviral (EEASPA) que avalia a dificuldade em seguir as prescrições para o tratamento em circunstâncias que exigem maior planejamento, atenção e organização para tomar a medicação. Essas situações difíceis tendem a diminuir a preocupação com a doença ou a confiança no tratamento. Geralmente, são experiências negativas com a medicação e os efeitos negativos que estão presentes nesse processo (Leite et al., 2002). Os valores calculados retornam médias entre 0 e 4 pontos, interpretadas como quanto maior a pontuação maior a expectativa de autoeficácia. $\mathrm{O}$ conteúdo dos itens presentes na escala sugere três situações difíceis para seguir a prescrição: a primeira (A) contempla 07 itens da Escala (5, 6, 7, 13, 16, 17, 19); a segunda (B) são 05 itens (1, 2 e 12, 20 e 8); e a terceira (C) contempla 09 itens (10, 15, 18, 21 além dos itens 3, 4, 11, 9 e 14) (Quadro 1 e Tabela 2).

Quadro 1: Descrição das situações relacionadas a EEASPA.

\begin{tabular}{|l|l|l|}
\hline \multicolumn{2}{|c|}{ SITUAÇÕES } & \multicolumn{1}{c|}{ DESCRIÇÃO } \\
\hline A & $\begin{array}{l}\text { Concentração e atividades } \\
\text { não rotineiras }\end{array}$ & $\begin{array}{l}\text { Exigem planejamento, atenção e organização para tomar a medicação. Referem-se à } \\
\text { distração, à falta dos medicamentos ou dos alimentos necessários para sua absorção } \\
\text { adequada. Esses itens correspondem às atividades que exigem maior concentração, } \\
\text { atividades não-rotineiras ou fora de casa e experiências sociais de lazer. }\end{array}$ \\
\hline B & $\begin{array}{l}\text { Preocupação com a doença e } \\
\text { o tratamento }\end{array}$ & $\begin{array}{l}\text { Tendem a diminuir a preocupação com a doença ou a confiança no tratamento. O que } \\
\text { diminuem a preocupação inclui a carga viral plasmática indetectável e o bom estado de } \\
\text { saúde. E, questões que afetam a confiança no tratamento como a descontinuidade no } \\
\text { atendimento clínico, estar com alguém que acha o tratamento desnecessário e o mau } \\
\text { estado de saúde. }\end{array}$ \\
\hline C & $\begin{array}{l}\text { Experiências negativas } \\
\text { Sbrangem experiências negativas com os ARV e afetos negativos de qualquer natureza. } \\
\text { medo da discriminação ou crítica social. }\end{array}$ \\
\hline
\end{tabular}


O banco de dados foi produzido no software Epi Info 7.0 e as análises executadas a partir do software SPSS 21.0. Correlacionaram-se os itens da EEASPA com o CEAT-VIH através do coeficiente de correlação de Spearman, dada a natureza assimétrica das variáveis. Considerou-se um nível de significância de 5\% para as comparações estabelecidas.

O projeto foi aprovado pelo Comitê de Ética em Pesquisa da Universidade Federal de Santa Maria tendo o parecer $n^{\circ}$ 1.538.216. O desenvolvimento do estudo atendeu às normas nacionais de ética em pesquisa envolvendo seres humanos, com uso de Termo de Consentimento Livre e Esclarecido anterior ao momento da coleta de dados.

\section{Resultados}

Os participantes do estudo eram $56,5 \%$ do sexo feminino, $64,3 \%$ de cor de pele branca, $50,6 \%$ conviviam com esposo(a) ou companheiro(a), 61,3\% eram desempregados, $87,5 \%$ não utilizavam drogas, $78,6 \%$ dos participantes referiam não ingerir bebidas alcoólicas e 57,5\% não relataram mudança no estilo de vida depois do diagnóstico. Além disso, a via de transmissão sexual ocorreu em 70,8\% dos participantes, $60,7 \%$ não apresentaram doença oportunista, $22,6 \%$ relataram uso de medicamento de atuação no sistema nervoso e 77,4\% apresentaram CV indetectável.

A adesão estrita estava presente em 33,7\% $(n=56)$ dos participantes, insuficiente para $65,1 \%(n=108)$ e inadequada em $1,2 \%(n=2)$. Na Tabela 1, encontra-se a distribuição das situações (A, B e C) da EEASPA quanto aos níveis de adesão classificadas como inadequada, insuficiente e estrita.

Tabela 1: Distribuição das situações de expectativa de autoeficácia quanto aos níveis de adesão, RS, Brasil, 2018.

\begin{tabular}{ccccccc}
\hline & \multicolumn{2}{c}{ Níveis de adesão } \\
\cline { 2 - 7 } & \multicolumn{2}{c}{ Inadequada } & \multicolumn{2}{c}{ Insuficiente } & \multicolumn{2}{c}{ Estrita } \\
\hline Situações & Média & Desvio Padrão & Média & Desvio Padrão & Média & Desvio Padrão \\
\hline A & 3,07 & 0,71 & 3,83 & 0,31 & 3,92 & 0,21 \\
B & 3,40 & 0,57 & 3,90 & 0,25 & 3,95 & 0,14 \\
C & 2,56 & 0,31 & 3,81 & 0,31 & 3,90 & 0,19 \\
\hline
\end{tabular}

Legenda: $\mathrm{A}$ = Concentração e atividades não rotineiras, $\mathrm{B}=$ Preocupação com a doença e o tratamento, $\mathrm{C}=$ Experiências negativas. Fonte: Autores.

Da mesma forma como observado na Tabela 1, as menores médias estão concentradas nas situações A e C, sendo que, as médias de todas as situações ficam menores conforme os níveis de adesão são mais baixos. Na situação A, os itens do instrumento que apresentaram menores médias foram "Se eu estiver na rua" ( $M=3,57$; DP 1,04) e "Se eu tiver de tomar remédios várias vezes por dia" $(\mathrm{M}=3,72 ; \mathrm{DP}=0,77)$. Sendo que os itens da situação $\mathrm{C}$ que se correlacionaram de forma estatisticamente significativa com a adesão global foram as questões 3, 4, 9, 10, 11 e 21 da EEASPA (Tabela 2). 
Tabela 2: Itens conforme a situação da Escala de Expectativa de Autoeficácia para Seguir Prescrição Antirretroviral (EEASPA) e correlação de Spearman entre os itens da situação C da e adesão global, RS, Brasil, 2018.

\begin{tabular}{|c|c|c|c|c|c|c|c|}
\hline & Itens da EEASPA & $\mathbf{M}$ & DP & Min & Max & $\mathbf{r}_{\mathbf{s}}$ & $\mathbf{p}$ \\
\hline \multirow{7}{*}{ 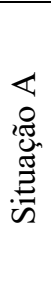 } & 5. Se eu estiver ocupado ou me divertindo. & 3,82 & 0,64 & 0 & 4 & & \\
\hline & 6. Se eu estiver em viagem de passeio ou de trabalho. & 3,92 & 0,39 & 1 & 4 & & \\
\hline & 7. Se eu estiver na rua. & 3,57 & 1,04 & 0 & 4 & & \\
\hline & 13. Se eu tiver de tomar remédios várias vezes por dia. & 3,72 & 0,77 & 0 & 4 & & \\
\hline & 16. Se for feriado ou final de semana. & 3,97 & 0,20 & 2 & 4 & & \\
\hline & 17. Se eu tiver de mudar meu horário de comer ou de dormir. & 3,90 & 0,44 & 1 & 4 & & \\
\hline & 19. Se eu estiver fazendo coisas fora da minha rotina. & 3,86 & 0,50 & 0 & 4 & & \\
\hline \multirow{5}{*}{ 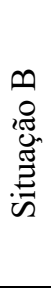 } & 8. Se eu estiver me sentindo doente. & 3,92 & 0,42 & 0 & 4 & & \\
\hline & 1. Se eu estiver bem de saúde. & 3,92 & 0,43 & 0 & 4 & & \\
\hline & $\begin{array}{l}\text { 2. Se o vírus no meu sangue for tão pouco que não aparece no exame } \\
\text { de carga viral. }\end{array}$ & 3,86 & 0,59 & 0 & 4 & & \\
\hline & 12. Se mudar muito o médico que me atende. & 3,84 & 0,59 & 0 & 4 & & \\
\hline & $\begin{array}{l}\text { 20. Se eu estiver com alguém que acha bobagem eu tomar esses } \\
\text { remédios. }\end{array}$ & 3,91 & 0,44 & 0 & 4 & & \\
\hline \multirow{9}{*}{ 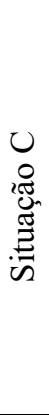 } & $\begin{array}{l}\text { 9. Se eu estiver com alguém que eu não quero que saiba que sou } \\
\text { portador do vírus da AIDS. }\end{array}$ & 3,69 & 0,98 & 0 & 4 & 0,17 & $\mathbf{0 , 0 3 1}$ \\
\hline & 10. Se eu tiver de tomar muitos comprimidos. & 3,69 & 0,82 & 0 & 4 & 0,25 & $\mathbf{0 , 0 0 1}$ \\
\hline & 11. Se eu estiver nervoso ou irritado. & 3,83 & 0,69 & 0 & 4 & 0,29 & $<0,001$ \\
\hline & 14. Se eu estiver com pessoas estranhas. & 3,75 & 0,83 & 0 & 4 & 0,13 & 0,091 \\
\hline & 15. Se o remédio for difícil de engolir. & 3,87 & 0,51 & 1 & 4 & 0,14 & 0,074 \\
\hline & 18. Se o remédio tiver gosto ruim ou cheiro forte. & 3,84 & 0,63 & 0 & 4 & 0,26 & $\mathbf{0 , 0 0 1}$ \\
\hline & 21. Se os remédios estiverem me causando efeito ruim. & 3,36 & 1,20 & 0 & 4 & 0,17 & $\mathbf{0 , 0 3 1}$ \\
\hline & 3. Se eu estiver aborrecido e me sentindo pra baixo. & 3,89 & 0,52 & 0 & 4 & 0,30 & $<\mathbf{0 , 0 0 1}$ \\
\hline & 4. Se eu for discriminado ou rejeitado. & 3,89 & 0,47 & 1 & 4 & 0,26 & 0,001 \\
\hline
\end{tabular}

Legenda: M: Média; DP: Desvio Padrão; Min: Mínimo; Max: Máximo; $\mathrm{r}_{\mathrm{s}}$ : Correlação de Spearman.

Fonte: adaptado de Leite et al. (2002).

As células de defesa T-CD4+, CV, cumprimento, antecedentes e adesão global apresentaram correlação significativa com a EEASPA. Para a as células T-CD4+, essa correlação foi direta e fraca. A CV também apresentou correlação fraca, no entanto, inversa. Cumprimento e antecedentes apresentaram correlação fraca e direta, ao passo que adesão global demonstrou correlação moderada e direta (Tabela 3).

Tabela 3: Correlação entre autoeficácia, indicadores clínicos e as dimensões de adesão do CEAT-VIH. RS, Brasil, 2018.

\begin{tabular}{lccc}
\hline Variáveis & Coeficiente de correlação & $\mathbf{p}$ & $\mathbf{N}$ \\
\hline Indicadores clínicos & & & \\
Células T-CD4+ & $0,212^{*}$ & $\mathbf{0 , 0 0 8}$ & 155 \\
Carga Viral & $-0,170^{*}$ & $\mathbf{0 , 0 3 2}$ & 159 \\
Adesão & & & \\
Cumprimento & $0,263^{*}$ & $\mathbf{0 , 0 0 1}$ & 166 \\
Antecedentes & $0,283^{*}$ & $\mathbf{0 , 0 0 0}$ & 166 \\
Comunicação & 0,108 & 0,166 & 166 \\
Crenças & 0,121 & 0,120 & 166 \\
Satisfação & 0,129 & 0,098 & 166 \\
Adesão global & $0,324^{* *}$ & $\mathbf{0 , 0 0 0}$ & 166 \\
\hline
\end{tabular}

Legenda: *correlação fraca; ** correlação moderada.

Fonte: Autores.

\section{Discussão}

Os resultados deste estudo evidenciam que as atividades não rotineiras, como viagem, passeio e lazer, estão associadas à baixa expectativa de autoeficácia e à consequente baixa adesão à TARV. Sair da rotina e estar fora de casa 
implica no convívio com as pessoas em sociedade que desenvolveu estigmas e preconceitos relacionados à infecção. Desse modo, amenizar as atitudes preconceituosas auxiliaria tanto na adesão como na expectativa de autoeficácia. Ao minimizar o estigma na sociedade, a pessoa que vive com o HIV não apresentaria receio caso alguém presenciasse a ingesta do medicamento (Lenzi et al., 2018). Dessa forma, as preocupações com o sigilo em relação ao diagnóstico da infecção são indicadores negativos de qualidade de vida (Primeira et al., 2020). As expectativas de autoeficácia diminuem em relação à adesão e ao seguimento da prescrição de TARV, a preocupação com o sigilo vem ao encontro da carga cultural negativa no que se refere à infecção pelo HIV.

As experiências negativas vivenciadas pelas pessoas que vivem com HIV implicam no seu estado de humor, como sentir-se triste e deprimido, e são reflexos da percepção estigmatizada e discriminatória advinda da sociedade. As situações que envolvem pessoas que não conhecem o diagnóstico daquele que vive com HIV são fatos que dificultam a ingestão dos ARV, seja pelo medo de ser visto ingerindo ou pelo medo de sofrer discriminação e exclusão do meio social no qual está inserido (Camacho et al., 2019; Rintamaki et al., 2019).

Estes fatores implicam no apoio que a pessoa que vive com HIV poderá receber ou não a partir do seu diagnóstico. Estudo evidenciou que há correlação positiva entre suporte emocional e situações que mostram falta de adesão antecedente, e que a adesão e o suporte social se correlacionam positivamente com a comunicação entre o médico e o paciente (Oliveira et al., 2020). Isso indica que, apesar de o tratamento atual ser de primeira linha, a consciência construída culturalmente sobre a infecção prejudica a EAE e a confiança em si mesmo para executar atividades benéficas em relação ao tratamento. Episódios de preconceito, seja por parte dos familiares ou profissionais da saúde, remetem a sentimentos de abandono e incapacidade de viver em sociedade, impactando na baixa crença de autoeficácia (Pires \& Santos, 2018).

Assim, entendemos que pessoas com HIV que vivenciam experiências negativas, tendem a diminuir a EAE para seguir a prescrição de ARV. Momentos de desagrado e de tristeza tendem a diminuir a expectativa de autoeficácia. A depressão em pessoas que vivem com HIV estimula a sensação de descrença no tratamento, pois esse sentimento ocasiona a negação em relação à infecção. Nesses momentos, diminui o grau de adesão aos ARV e induz a adoção de estilo de vida não saudável, como o uso abusivo de álcool e outras drogas (Silva et al., 2017). Então, verifica-se que, com relação às pessoas desmotivadas com o tratamento, as pessoas que vivenciam esse processo com motivação tendem a obter níveis de autoeficácia maiores.

Ao estudar os processos psicossociais da resiliência entre as pessoas que vivem com HIV, o suporte social foi um fator importante que implicou positivamente na expectativa de autoeficácia para seguir a prescrição de ARV, de tal forma que, quando a pessoa que vive com HIV recebe apoio emocional das pessoas próximas e no ambiente de trabalho, a adesão ao tratamento é eficaz. Sentir-se acolhido em ambientes sociais estimula o seguimento do tratamento e da prescrição, ao contrário de ambientes desfavoráveis, nos quais o estigma e o preconceito estão presentes e podem diminuir a expectativa de autoeficácia e crença no tratamento da infecção (Araújo et al., 2019).

Houve situações em que a pessoa que vive com HIV concordou que quando sentia nervosismo e irritabilidade a expectativa de autoeficácia diminuía. A associação entre o abandono da TARV está relacionada com a presença de transtornos psiquiátricos, dificuldades para comparecer ao serviço de saúde e ausência de suporte social afetivo (Granada et al., 2018; Mello et al., 2020).

Os fatores de saúde mental mais relevantes associados à não adesão à TARV são o uso de álcool, depressão e o estigma (Carvalho et al., 2020). Isso poderá ser alusivo à discriminação e ao estigma social. Por outro lado, em ambientes acolhedores e afetuosos, esses sentimentos não são vivenciados e a pessoa se sente acolhida e bem aceita nesse convívio social. A rotina de conviver com pessoas que aceitam o diagnóstico e de viver outras situações de sentimentos positivos aumenta as expectativas de autoeficácia para seguir o tratamento e aderir de forma efetiva à prescrição (Calvetti et al., 2016, Nogueira \& 
Seidl, 2016).

As expectativas negativas com os ARV em relação ao gosto, cheiro desagradável, efeitos colaterais presentes no início do tratamento e número de comprimidos administrados ao dia são preditores de baixa autoeficácia. A necessidade de ingerir medicamentos em mais de um horário durante o dia ou de alimentação prévia para prevenir efeitos colaterais exige organização de horário e disciplina (Silva et al., 2017). As situações que precisam de uma organização prévia demandam tempo e resiliência dessas pessoas (Araújo et al., 2019).

Ainda nessa perspectiva, em que a mudança da rotina diária impacta na ingesta dos ARV, momentos em que a pessoa precisa se ausentar da sua rotina e modificar o horário de administração dos medicamentos, potencializa a irregularidade do tratamento e a organização prévia precisa ser ajustada (Zuge et al., 2020). Caso o medicamento apresente cheiro e gosto ruim, a pessoa tende a não administrar diariamente devido ao desconforto que essas experiências representam na rotina $\mathrm{e}$, principalmente, na hora da tomada dos medicamentos (Costa \& Meirelles, 2019). Os efeitos adversos relacionados ao uso dos ARV refletiram negativamente a expectativa de autoeficácia para adesão. As baixas expectativas de eficácia para adesão ao TARV foram mais comuns nas experiências de efeitos adversos do tratamento. Essas situações são preditores de adesão e, quanto maior os efeitos colaterais, menores são as expectativas de autoeficácia para seguir com a prescrição (Drachler et al., 2016).

\section{Considerações Finais}

Os resultados relacionados às situações que se referem às experiências negativas com o uso dos ARVs e, também, aos afetos negativos de qualquer natureza vivenciados nas relações sociais, apresentaram as menores médias e maior correlação, mesmo que fraca, entre a EAE e adesão global. Entendemos que este estudo poderá contribuir para reafirmar a necessidade de desconstrução do estigma social que as pessoas que vivem com HIV experimentam e de ampliação do enfoque da adesão de modo que não se restrinja aos aspectos clínicos.

\section{Agradecimentos}

Agradecemos à todas as pessoas que vivem com HIV que participaram desta pesquisa, aos bolsistas de iniciação científica que contribuíram para os resultados deste estudo, à Universidade Federal de Santa Maria e ao Hospital Universitário de Santa Maria pela estrutura cedida. O presente trabalho foi realizado com apoio da Coordenação de Aperfeiçoamento de Pessoal de Nível Superior - Brasil (CAPES) - Código de Financiamento 001 e do Conselho Nacional de Desenvolvimento Científico e Tecnológico (CNPq), Edital Universal CNPq 01/2016: Processo nº 408709/2016-2.

\section{Referências}

Amirkhanian, Y. A., Kelly, J. A., DiFranceisco, W. J., Kuznetsova, A. v., Tarima, S. S., Yakovlev, A. A., \& Musatov, V. B. (2018). Predictors of HIV Care Engagement, Antiretroviral Medication Adherence, and Viral Suppression Among People Living with HIV Infection in St. Petersburg, Russia. AIDS and Behavior, 22(3). https://doi.org/10.1007/s10461-016-1638-9

Araújo, L. F. de, Leal, B. de S., Santos, J. V. de O., \& Sampaio, A. V. C. (2019). Análise da Resiliência entre Pessoas que Vivem com HIV/AIDS: Um Estudo Psicossocial. Psicologia: Teoria e Pesquisa, 35. https://doi.org/10.1590/0102.3772e35416

Bandura, A. (1977). Self-efficacy: Toward a unifying theory of behavioral change. Psychological Review, 84(2). https://doi.org/10.1037/0033-295X.84.2.191 Calvetti, P. Ü., Giovelli, G. R. M., Gauer, G. J. C., \& Moraes, J. F. D. de. (2016). Níveis de Ansiedade, Estresse Percebido e Suporte Social em Pessoas que Vivem com HIV/Aids. Psicologia: Teoria e Pesquisa, 32(4). https://doi.org/10.1590/0102.3772e324317

Camacho, G., Kalichman, S., \& Katner, H. (2019). Anticipated HIV-Related Stigma and HIV Treatment Adherence: The Indirect Effect of Medication Concerns. AIDS and Behavior 2019 24:1, 24(1), 185-191. https://doi.org/10.1007/S10461-019-02644-Z

Cardoso, T. S., Costa, J. de O., Reis, E. A., Silveira, M. R., Bonolo, P. de F., Santos, S. F. dos, \& Ceccato, M. das G. B. (2019). Which antiretroviral regimen is associated with higher adherence in Brazil? A comparison of single, multi, and dolutegravir-based regimens. Cadernos de Saúde Pública, 35(9). https://doi.org/10.1590/0102-311x00115518 
Carvalho, P. M. de M., Monteiro da Silva Neto, R., \& Leite Rolim Neto, M. (2020). Fatores de saúde mental associados à não adesão à terapia anti-retroviral: uma revisão sistemática. Saúde Coletiva (Barueri), 57. https://doi.org/10.36489/saudecoletiva.2020v10i57p3665-3690

Costa, V. T., \& Meirelles, B. H. S. (2019). Adherence to treatment of young adults living with HIV/Aids from the perspective of complex thinking. Texto \& Contexto - Enfermagem, 28. https://doi.org/10.1590/1980-265x-tce-2017-0016

Dave, S., Peter, T., Fogarty, C., Karatzas, N., Belinsky, N., \& Pant Pai, N. (2019). Which community-based HIV initiatives are effective in achieving UNAIDS 90-90-90 targets? A systematic review and meta-analysis of evidence (2007-2018). PLOS ONE, 14(7). https://doi.org/10.1371/journal.pone.0219826

Drachler, M. de L., Drachler, C. W., Teixeira, L. B., \& de Carvalho Leite, J. C. (2016). The Scale of Self-Efficacy Expectations of Adherence to Antiretroviral Treatment: A Tool for Identifying Risk for Non-Adherence to Treatment for HIV. PLOS ONE, 11(2). https://doi.org/10.1371/journal.pone.0147443

Granada, A. M., Vanegas, C., Forero, E., Silva, C., \& Vergara, E. P. (2018). Factors associated with the abandonment of highly effective antiretroviral therapy in patients with HIV in a third-level hospital. Acta Medica Colombiana, 43(1), 31-36.

Leite, J. C. de C., Drachler, M. de L., Centeno, M. O., Pinheiro, C. A. T., \& Silveira, V. L. da. (2002). Desenvolvimento de uma escala de auto-eficácia para adesão ao tratamento anti-retroviral. Psicologia: Reflexão e Crítica, 15(1). https://doi.org/10.1590/S0102-79722002000100014

Lenzi, L., Tonin, F. S., Souza, V. R. de, \& Pontarolo, R. (2018). Suporte Social e HIV: Relações Entre Características Clínicas, Sociodemográficas e Adesão ao Tratamento. Psicologia: Teoria e Pesquisa, 34(0). https://doi.org/10.1590/0102.3772e34422

Loch, A. P., Caraciolo, J. M. de M., Rocha, S. Q., Fonsi, M., Souza, R. de A., Gianna, M. C., Gonçalves, A., \& Kalichman, A. O. (2020). Intervenção para a implementação do monitoramento clínico em serviços especializados de atenção às pessoas vivendo com HIV/aids. Cadernos de Saúde Pública, 36(5). https://doi.org/10.1590/0102-311x00136219

Mello, C. J. F. de A., Amaral, J. C. S. do, Costa, M. da S., Cavalcante, M. de N. M., Rêgo, N. M. de S., Silva, L. M. R. da, \& Amaral, R. R. do. (2020). Terapia Antirretroviral: principais causas de abandono no estado do Amapá. Revista Eletrônica Acervo Saúde, 12(8). https://doi.org/10.25248/reas.e3423.2020

Musayón-Oblitas, F. Y., Cárcamo, C. P., Gimbel, S., Zarate, J. I. E., \& Espinoza, A. B. G. (2020). Validation of a counseling guide for adherence to antiretroviral therapy using implementation science. Revista Latino-Americana de Enfermagem, 28. https://doi.org/10.1590/1518-8345.3117.3228

Nogueira, G. S., \& Seidl, E. M. F. (2016). Associação entre percepção de doença e ansiedade, depressão e autoeficácia em pessoas com HIV/Aids. Temas Em Psicologia, 24(2). https://doi.org/10.9788/TP2016.2-12

Oliveira, R. da S., Primeira, M. R., Santos, W. M. dos, Paula, C. C. de, \& Padoin, S. M. de M. (2020). Association between social support and adherence to anti-retroviral treatment in people living with HIV. Revista Gaúcha de Enfermagem, 41. https://doi.org/10.1590/1983-1447.2020.20190290

Pires, C. O., \& Santos, C. (2018). Qualidade de vida do paciente frente ao diagnóstico de HIV/Aids. Revista Da Mostra de Trabalhos de Conclusão de Cruso TCC - Congrega, 684-693.

Primeira, M. R., Santos, W. M. dos, Paula, C. C., \& Padoin, S. M. de M. (2020). Quality of life, adherence and clinical indicators among people living with HIV. ACTA Paulista de Enfermagem, 33. https://doi.org/10.37689/ACTA-APE/2020AO0141

Remor, E., Milner-Moskovics, J., \& Preussler, G. (2007). Adaptação brasileira do “Cuestionario para la Evaluación de la Adhesión al Tratamiento Antiretroviral.” Revista de Saúde Pública, 41(5). https://doi.org/10.1590/S0034-89102006005000043

Rintamaki, L., Kosenko, K., Hogan, T., Scott, A. M., Dobmeier, C., Tingue, E., \& Peek, D. (2019). The Role of Stigma Management in HIV Treatment Adherence. International Journal of Environmental Research and Public Health 2019, Vol. 16, Page 5003, 16(24), 5003. https://doi.org/10.3390/IJERPH16245003

Santos, K. R. F. do N., Carneiro, W. S., Vieira, A. da S., Souza, M. B., \& Gonçalves, C. F. G. (2020). Fatores que interferem na adesão e permanência da terapia antirretroviral. Brazilian Journal of Health Review, 3(2). https://doi.org/10.34119/bjhrv3n2-143

Seghatol-Eslami, V. C., Dark, H. E., Raper, J. L., Mugavero, M. J., Turan, J. M., \& Turan, B. (2017). Brief Report: Interpersonal and Intrapersonal Factors as Parallel Independent Mediators in the Association Between Internalized HIV Stigma and ART Adherence. JAIDS Journal of Acquired Immune Deficiency Syndromes, 74(1). https://doi.org/10.1097/QAI.0000000000001177

Silva, R. A. R. da, Costa, M. M. do N., Souza Neto, V. L. de, Silva, B. C. O. da, Costa, C. da S., \& Andrade, I. F. C. de. (2017). Noncompliance in people living with HIV: accuracy of defining characteristics of the nursing diagnosis. Revista Latino-Americana de Enfermagem, 25(0). https://doi.org/10.1590/15188345.1582 .2940

Souza, H. C. de, Mota, M. R., Alves, A. R., Lima, F. D., Chaves, S. N., Dantas, R. A. E., Abdelmur, S. B. M., \& Mota, A. P. V. da S. (2019). Analysis of compliance to antiretroviral treatment among patients with HIV/AIDS. Revista Brasileira de Enfermagem, 72(5). https://doi.org/10.1590/0034-7167-20180115

Velame, K. T., Silva, R. de S. da, \& Cerutti Junior, C. (2020). Factors related to adherence to antiretroviral treatment in a specialized care facility. Revista Da Associação Médica Brasileira, 66(3). https://doi.org/10.1590/1806-9282.66.3.290

Zhang, L., Li, X., Lin, Z., Jacques-Tiura, A. J., Xu, J., Zhou, Y., Qiao, S., Shen, Z., \& Stanton, B. (2016). Side effects, adherence self-efficacy, and adherence to antiretroviral treatment: a mediation analysis in a Chinese sample. AIDS Care, 28(7). https://doi.org/10.1080/09540121.2015.1124984

Zuge, S. S., Paula, C. C. de, \& Padoin, S. M. de M. (2020). Efetividade de intervenções para adesão à terapia antirretroviral em adultos com HIV: revisão sistemática. Revista da Escola de Enfermagem da USP, 54. https://doi.org/10.1590/s1980-220x2019009803627 